\title{
REVISED
}

\section{Unusual C25 Steroids Produced by a Sponge-Derived Penicillium citrinum}

Taro Amagata, ${ }^{\dagger}$ Akiko Amagata, ${ }^{\dagger}$ Karen Tenney, ${ }^{\dagger}$ Frederick A. Valeriote, ${ }^{\dagger}$ Emil Lobkovsky, ${ }^{\S}$

$$
\text { Jon Clardy, }{ }^{\mathbb{T}} \text { and Phillip Crews }{ }^{*},{ }^{\dagger}
$$

[salicitrins SI ver.5_Org. Lett. 09/26/03]

${ }^{\dagger}$ Department of Chemistry and Biochemistry and Institute for Marine Sciences, University of California Santa Cruz, Santa Cruz, California 95064

*Josephine Ford Cancer Center, Detroit, Michigan 48202

${ }^{\S}$ Department of Chemistry and Chemical Biology, Cornell University, Ithaca, New York 14853

${ }^{I I}$ Department of Biological Chemistry and Molecular Pharmacology, Harvard Medical School, Boston, MA 02115

phil@chemistry.ucsc.edu

\section{[Contents]}

Experimental Procedure

Figure S1. ${ }^{1} \mathrm{H}$ NMR spectrum of $\mathbf{1}$

Figure S2. ${ }^{13} \mathrm{C}$ NMR spectrum of $\mathbf{1}$

Figure S3. ${ }^{1} \mathrm{H}$ NMR spectrum of $\mathbf{2}$

Figure S4. ${ }^{13} \mathrm{C}$ NMR spectrum of 2

Table S1. Comparison of ${ }^{13} \mathrm{C}$ NMR data between isocyclocitrinol A(1) and revised structure of cyclocitrinol (3a)

Table S2. ${ }^{1} \mathrm{H}$ and ${ }^{13} \mathrm{C}$ NMR data of 2

Table S3. Crystal data and structure refinement for 2

Table S4. Atomic coordinates $\left(\times 10^{4}\right)$ and equivalent isotropic displacement parameters $\left(\AA^{2} \times 10^{3}\right)$ for 2

Table S5. Bond lengths $[\AA]$ and angles $\left[{ }^{\circ}\right]$ for 2

Table S6. Anisotropic displacement parameters $\left(\AA^{2} \times 10^{3}\right)$ for 2

Table S7. Hydrogen coordinates $\left(\times 10^{4}\right)$ and isotropic displacement parameters $\left(\AA^{2} \times 10^{3}\right)$ for 2 


\section{Experimental Procedure}

General Experimental Procedures. The NMR spectra were recorded at 500 and 125.7 $\mathrm{MHz}$ for ${ }^{1} \mathrm{H}$ and ${ }^{13} \mathrm{C}$, respectively. UV/vis measurements were performed with a diode array detector. HPLC was performed with columns of $4 \mu \mathrm{m}$ ODS.

Biological Materials. The fungus (strain no. 991084) was isolated from an Axinellida sponge (coll. no. 99108) collected using SCUBA off the coast of Papua New Guinea on December 1999 by the same techniques described previously ${ }^{1}$. The strain was identified as a Penicillium citrinum based on the analyses of both the 25S ribosomal DNA sequence and its fruiting body. This fungus is maintained in a cryopreserved state at UCSC.

Culture conditions. The fungal strain was grown in a liquid medium $(20 \mathrm{~L})$ containing 3.5\% Czapek-Dox broth and 0.5\% yeast extract in filtered Monterey Bay seawater adjusted to $\mathrm{pH} 7.4$ at $180 \mathrm{rpm}$ for 28 days at room temperature $\left(25^{\circ} \mathrm{C}\right)$.

Disk Diffusion Soft Agar Colony Formation Assay. An in vitro cell-based assay was employed to identify solid tumor selectivity for original extracts, extract partition fractions, and pure compounds. The differential cytotoxicity is expressed by observing a zone differential between any solid tumor cell (Colon38, ColonH116, Lung H125) and either leukemia cells (L1210 or CEM) or normal cells (CFU-GM). The sample is designated as "solid tumor selective" if the zone units of solid tumor - normal cell or leukemia cells is greater than 250 units.

Extraction and Isolation. The culture was filtered under suction and the broth was extracted with equal volumes of EtOAc three times. The combined extract was evaporated to give a crude extract (465 mg). The organic extract was applied on reversed phase HPLC with $\mathrm{MeOH}-\mathrm{H}_{2} \mathrm{O}$ (1:1 to 1:0) to give 16 fractions. Fraction 9 and 15 were purified by reversed phase HPLC to afford 1 (3.8 $\mathrm{mg}$ ) and $\mathbf{2}(15.4 \mathrm{mg})$, respectively.

Isocyclocitrinol A (1). Obtained as an amorphous powder. $[\alpha]_{D}+136.0\left(c 0.05, \mathrm{CHCl}_{3}\right)$; $\lambda_{\max }(\mathrm{MeOH}) / \mathrm{nm} 243\left(\log \varepsilon\right.$ 3.94); ${ }^{1} \mathrm{H}$ and ${ }^{13} \mathrm{C}$ NMR data are listed in Table 1; HRESI-TOF-MS: $m / z$ 401.2682 [M+H] $]^{+}$(calcd for $\mathrm{C}_{25} \mathrm{H}_{37} \mathrm{O}_{4}, 401.2686$ ).

22-Acetylisocyclocitrinol A (2). Colorless crystals from MeOH. Mp. 209-210 ${ }^{\circ} \mathrm{C} .[\alpha]_{\mathrm{D}}$ $+128.0\left(\right.$ c $\left.0.15, \mathrm{CHCl}_{3}\right) ; \lambda_{\max }(\mathrm{MeOH}) / \mathrm{nm} 243\left(\log \varepsilon\right.$ 3.88); ${ }^{1} \mathrm{H}$ and ${ }^{13} \mathrm{C}$ NMR data are listed in Table S1; HRESI-TOF-MS: $m / z 443.2790[\mathrm{M}+\mathrm{H}]^{+}$(calcd for $\mathrm{C}_{27} \mathrm{H}_{39} \mathrm{O}_{5}, 443.2792$ ).

X-ray crystallography of 2 . The single crystal X-ray analysis was conducted as follows. Suitable crystals were obtained from $\mathrm{MeOH}$ by the vapor diffusion method. This crystal $(0.40$ x 0.30 x $0.20 \mathrm{~mm}^{3}$ ) was mounted on a Bruker SMART diffractometer (MoKo; $\left.-100^{\circ} \mathrm{C}\right)$. A 
hemisphere of data was taken using a narrow-scan routine (1406 frames, $0.3^{\circ}$ steps $\omega$-scan, exposure time was $30 \mathrm{~s} /$ frame, $2 \theta_{\max }=49.42^{\circ}$ ). Raw data was integrated with the Bruker SAINT + program $^{2}$ to yield a total 11733 reflections, of which 6914 were independent $\left(\mathrm{R}_{\mathrm{int}}=\right.$ $2.09 \%$, completeness $91.8 \%$ ) and 1793 with $\mathrm{I}>2 \sigma(\mathrm{I})$. Data were collected for absorption using SADABS program (min. and max. transmission are 0.9671 and 0.9833 , respectively). ${ }^{3}$ The structure was solved by direct methods and refined by full matrix least square on $\mathrm{F}^{2}$ techniques using anisotropic displacement parameters for all non-hydrogen atoms. ${ }^{4}$ All hydrogen atoms were found in the difference Fourier map and refine isotropically. At final convergence, $\mathrm{R}_{1}=4.70 \%$ and $\mathrm{GOF}=1.144$ for 441 parameters. Additional information about these data includes crystal data and structure refinement in Table S2, atomic coordinates in Table S3, bond length and angles in Table S4, anisotropic displacement parameters in Table S5, hydrogen coordinates in Table S6.

Alkaline hydrolysis of 2. To a solution of $2(3.8 \mathrm{mg})$ in $\mathrm{MeOH}(1.0 \mathrm{~mL})$, ammonium hydoxide $(600 \mu \mathrm{L})$ was added and left at $50^{\circ} \mathrm{C}$ for $3 \mathrm{~h}$. The reaction mixture was evaporated in a vacuum under reduced pressure to give a residue that was purified by a reversed phase HPLC using $\mathrm{MeCN}-\mathrm{H}_{2} \mathrm{O}(13: 7)$ to afford $1(2.0 \mathrm{mg})$. The ${ }^{1} \mathrm{H}$ and ${ }^{13} \mathrm{C}$ NMR spectra and the optical rotation $\left([\alpha]^{26}{ }_{\mathrm{D}}+110.0^{\circ} ; c 0.04, \mathrm{CHCl}_{3}\right)$ of the reaction product were identical with those of $\mathbf{1}$ isolated from the fungal extract. HRESI-TOF-MS: $m / z, 401.2681[\mathrm{M}+\mathrm{H}]^{+}$(calcd for $\mathrm{C}_{25} \mathrm{H}_{37} \mathrm{O}_{3}$, 401.2686).

Formation of the $(R)$ - and $(S)$-MTPA esters (4a and $4 b$ ) from 2. $(R)$-MTPA (15 mg), dicyclohexylcarbodiimide (20 $\mathrm{mg}$ ) and 4-(dimethylamino)pyridine (10 $\mathrm{mg})$ were added to a $\mathrm{CH}_{2} \mathrm{Cl}_{2}$ solution $(1 \mathrm{~mL})$ of $2(3.5 \mathrm{mg})$, and the reaction mixture was left at room temperature overnight. The solvent was evaporated in a vacuum under reduced pressure and the $\mathrm{MeOH}$ soluble portion of the residue was purified by a reversed phase HPLC using $\mathrm{MeOH}-\mathrm{H}_{2} \mathrm{O}$ (4:1) to afford ester $\mathbf{4 a}(2.2 \mathrm{mg})$. The same reaction with $2(5.5 \mathrm{mg})$ using $(S)$-MTPA $(10 \mathrm{mg})$ gave ester $\mathbf{4 b}(2.7 \mathrm{mg})$.

(R)-MTPA ester (4a). Obtained as white powder. ${ }^{1} \mathrm{H} \mathrm{NMR}\left(\mathrm{CDCl}_{3}\right) \delta 0.87(3 \mathrm{H}, \mathrm{s}$, $\mathrm{CH}_{3}-21$ ), 1.34 (3H, s, $\left.\mathrm{CH}_{3}-19\right), 1.47-1.67$ (4H, m, H11 $\left.\alpha, \mathrm{H} 12 \alpha, \mathrm{H} 15 \alpha, 15 \beta\right), 1.74-1.96$ (4H, m, $\mathrm{H} 11 \beta, \mathrm{H} 16 \alpha, \mathrm{H} 16 \beta, \mathrm{H} 17), 1.76$ (3H, d, J=6.3 Hz, $\left.\mathrm{CH}_{3}-25\right), 1.81$ (1H, m, H4ß), 2.10 (3H, s, $\left.\mathrm{COCH}_{3}\right), 2.13$ (1H, ddd, $\left.J=12.3,6.4,2.5 \mathrm{~Hz}, \mathrm{H} 14\right), 2.19$ (1H, m, H12ß), 2.41 (1H, m, H2 $\alpha$ ), 2.58 (1H, d, J=13.7 Hz, H18a), 2.63 (1H, m, H2ß), 2.64 (1H, dd, J=13.7, 6.2Hz, H18b), 2.79 (1H, m, H5), 2.84 (1H, dd, $J=12.4,5.7 \mathrm{~Hz}, \mathrm{H} 9), 2.89$ (1H, brd, $J=12.9 \mathrm{~Hz}, \mathrm{H} 4 \alpha$ ), 3.57 (3H, s, OMe), 4.84 (1H, brt, $J=11.4$ Hz, H3), 5.10 (1H, d, $J=7.3$ Hz, H22), 5.46 (1H, ddq, $J=15.2,6.7$, $1.4 \mathrm{~Hz}, \mathrm{H} 23), 5.63$ (1H, dd, J=7.8, $6.4 \mathrm{~Hz}, \mathrm{H} 1), 5.64(1 \mathrm{H}, \mathrm{s}, \mathrm{H} 7), 5.83(1 \mathrm{H}, \mathrm{dq}, J=15.1,6.7 \mathrm{~Hz}$, 
H24), 7.42 (3H, m, ArH), 7.56 (2H, m, ArH); HRESI-TOF-MS: $m / z 681.3027[\mathrm{M}+\mathrm{Na}]^{+}$(calcd $^{2}$ for $\mathrm{C}_{37} \mathrm{H}_{45} \mathrm{~F}_{3} \mathrm{O}_{7} \mathrm{Na}, 681.3010$ ).

(S)-MTPA ester (4b). Obtained as white powder. ${ }^{1} \mathrm{H}$ NMR $\left(\mathrm{CDCl}_{3}\right) \delta 0.86(3 \mathrm{H}, \mathrm{s}$, $\left.\mathrm{CH}_{3}-19\right), 1.33$ (3H, s, $\mathrm{CH}_{3}-21$ ), 1.48-1.67 (4H, m, H11 $\left.\alpha, \mathrm{H} 12 \alpha, \mathrm{H} 15 \alpha, 15 \beta\right), 1.74-1.97$ (4H, m, $\mathrm{H} 11 \beta, \mathrm{H} 16 \alpha, \mathrm{H} 16 \beta, \mathrm{H} 17), 1.77$ (3H, d, J=6.3 Hz, $\left.\mathrm{CH}_{3}-25\right), 1.86$ (1H, m, H4ß), 2.10 (3H, s, $\left.\mathrm{COCH}_{3}\right), 2.14$ (1H, ddd, J=12.3, 6.4, $\left.2.5 \mathrm{~Hz}, \mathrm{H} 14\right), 2.19$ (1H, m, H12ß), 2.31 (1H, m, H2 $\alpha$ ), 2.53 (1H, m, H2ß), 2.56 (1H, d, J=13.7 Hz, H18a), 2.64 (1H, dd, J=13.7, 6.3 Hz, H18b), 2.81 (1H, m, H5), 2.84 (1H, dd, $J=12.2,5.8 \mathrm{~Hz}, \mathrm{H} 9), 2.96$ (1H, brd, $J=12.9 \mathrm{~Hz}, \mathrm{H} 4 \alpha), 3.58$ (3H, s, OMe), 4.85 (1H, brt, $J=11.4$ Hz, H3), 5.09 (1H, d, $J=7.3 \mathrm{~Hz}, \mathrm{H} 22), 5.46$ (1H, ddq, $J=15.2,6.7$, $1.4 \mathrm{~Hz}, \mathrm{H} 23), 5.62$ (1H, dd, J=7.8, $6.4 \mathrm{~Hz}, \mathrm{H} 1), 5.64(1 \mathrm{H}, \mathrm{s}, \mathrm{H} 7), 5.83$ (1H, dq, J=15.1, $6.7 \mathrm{~Hz}$, H24), 7.41 (3H, m, ArH), 7.54 (2H, m, ArH). HRESI-TOF-MS: m/z $681.3020[\mathrm{M}+\mathrm{H}]^{+}$(calcd for $\mathrm{C}_{37} \mathrm{H}_{45} \mathrm{~F}_{3} \mathrm{O}_{7} \mathrm{Na}, 681.3010$ ). 


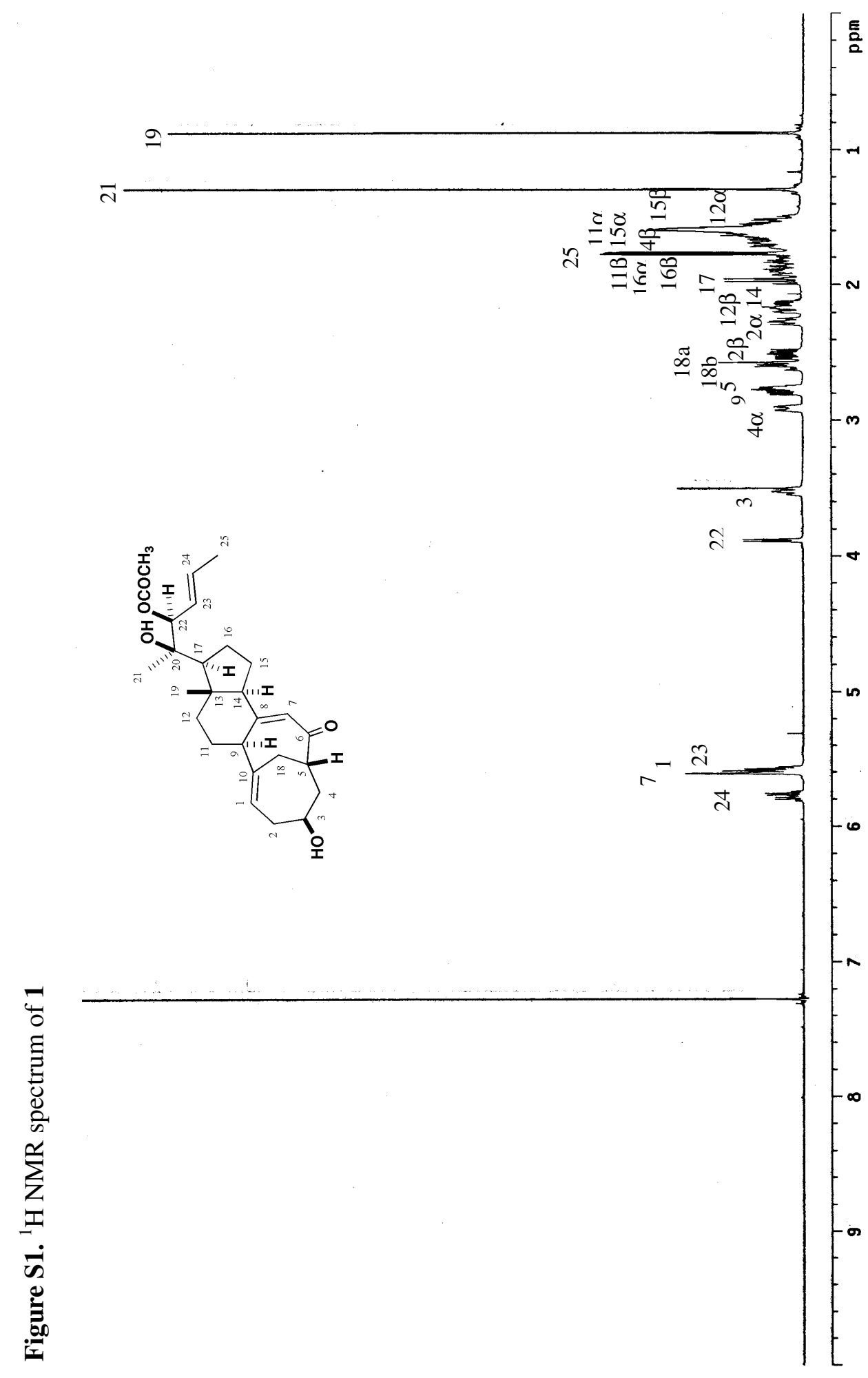




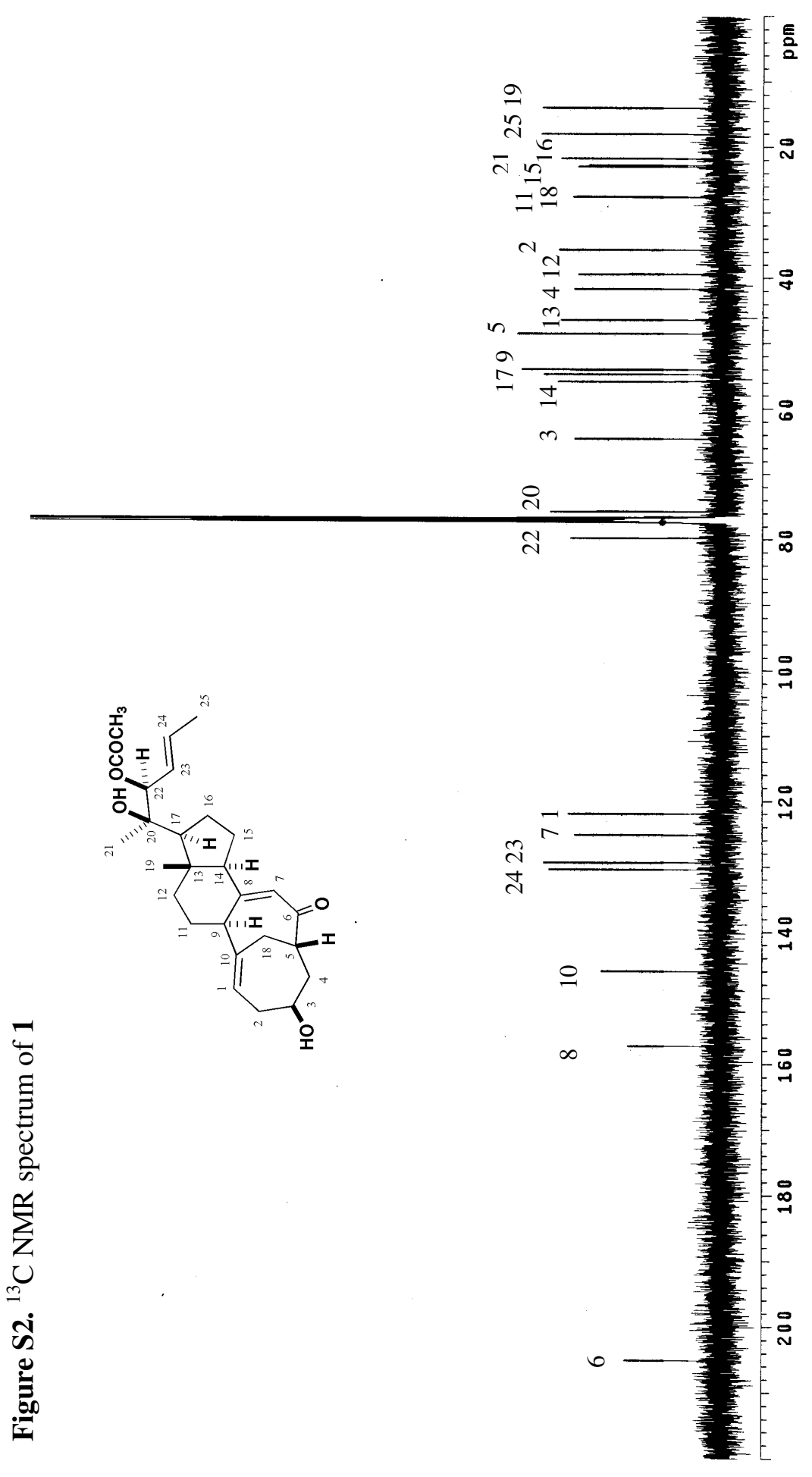




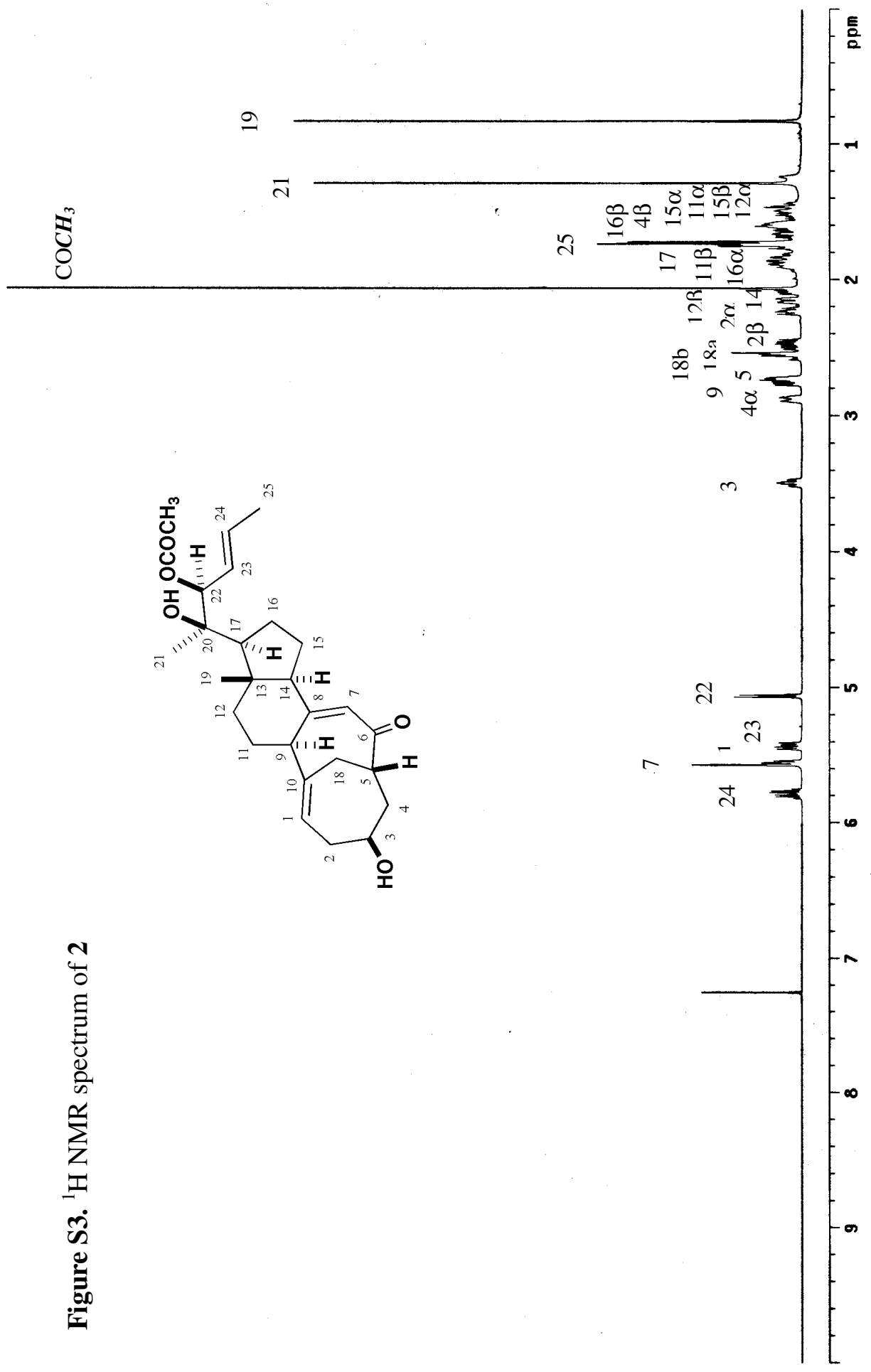




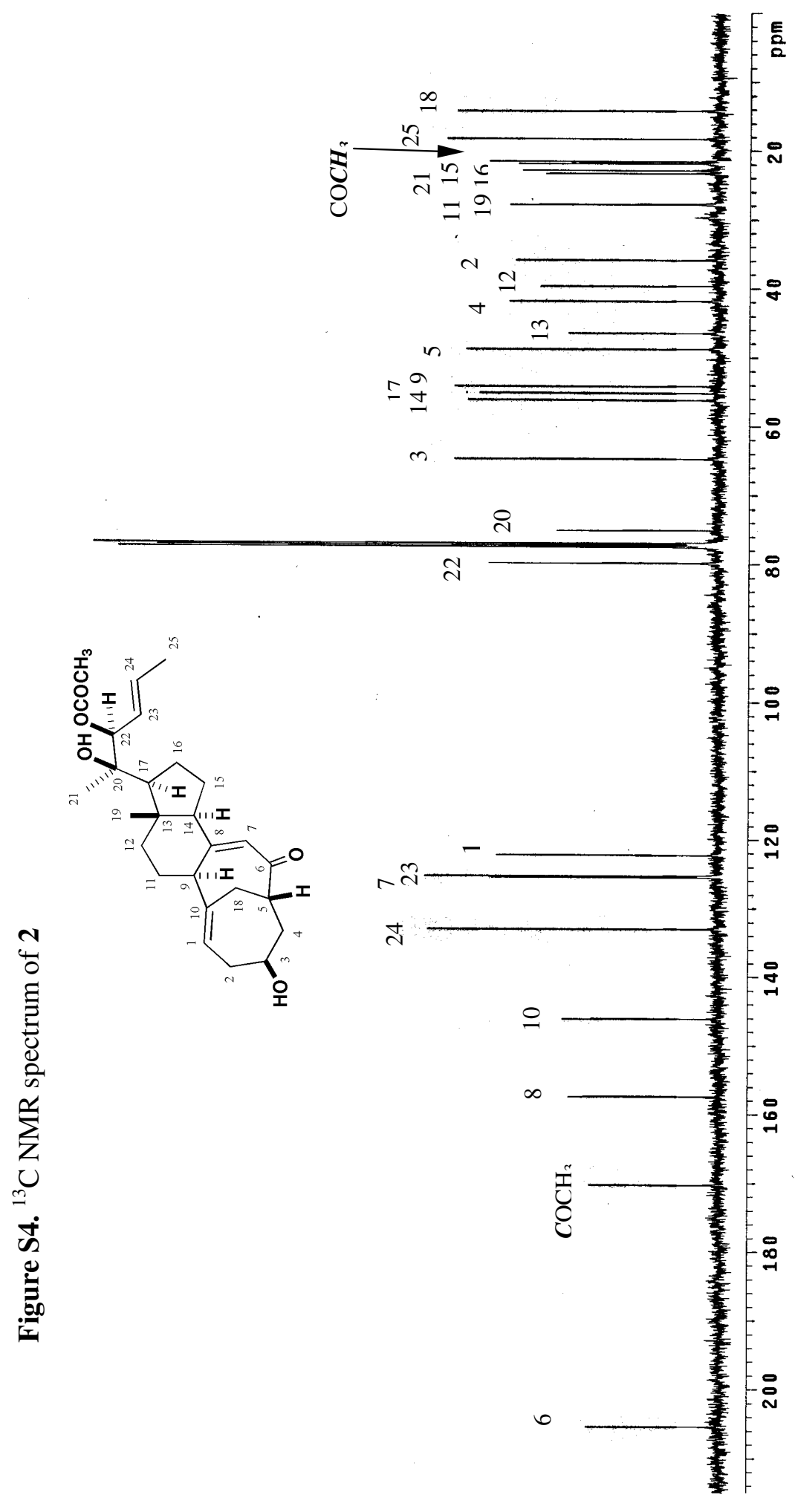




\begin{tabular}{|c|c|c|}
\hline Josition & 1 & $3 \mathrm{a}^{\mathrm{a}}$ \\
\hline 1 & 121.9 (d) & $122.2(\mathrm{~d})$ \\
\hline 2 & $35.7(\mathrm{t})$ & $36.2(\mathrm{t})$ \\
\hline 3 & $64.6(d)$ & 63.3 (d) \\
\hline 4 & $41.7(\mathrm{t})$ & $41.6(\mathrm{t})$ \\
\hline 5 & 48.5 (d) & 48.3 (d) \\
\hline 6 & 205.1 (s) & 204.3 (s) \\
\hline 7 & $125.2(d)$ & $124.7(\mathrm{~d})$ \\
\hline 8 & $157.3(\mathrm{~s})$ & $157.3(\mathrm{~s})$ \\
\hline 9 & $54.0(\mathrm{~d})$ & $53.5(\mathrm{~d})$ \\
\hline 10 & $146.0(\mathrm{~s})$ & $145.9(\mathrm{~s})$ \\
\hline 11 & $27.7(\mathrm{t})$ & $27.4(t)$ \\
\hline 12 & $39.4(\mathrm{t})$ & $39.0(\mathrm{t})$ \\
\hline 13 & $46.4(\mathrm{~s})$ & $46.1(\mathrm{~s})$ \\
\hline 14 & $55.8(\mathrm{~d})$ & 55.5 (d) \\
\hline 15 & $22.8(\mathrm{t})$ & $22.5(\mathrm{t})$ \\
\hline 16 & $21.8(\mathrm{t})$ & $22.3(\mathrm{t})$ \\
\hline 17 & 54.7 (d) & 60.1 (d) \\
\hline 18 & $27.6(t)$ & $27.7(\mathrm{t})$ \\
\hline 19 & $14.0(\mathrm{q})$ & $14.5(q)$ \\
\hline 20 & 75.7 (s) & $73.5(\mathrm{~s})$ \\
\hline 21 & $23.0(\mathrm{q})$ & $29.1(q)$ \\
\hline 22 & 79.8 (d) & 136.2 (d) \\
\hline 23 & 129.4 (d) & 131.2 (d) \\
\hline 24 & $130.4(d)$ & $66.5(d)$ \\
\hline 25 & $18.0(\mathrm{q})$ & $24.2(q)$ \\
\hline \multicolumn{3}{|c|}{ Data from the literature (see Figure } \\
\hline $\begin{array}{l}\text { 1), origi } \\
\text { and our }\end{array}$ & $\begin{array}{l}\text { atom num } \\
\text { ised numb }\end{array}$ & $\begin{array}{l}\text { ng of } \mathbf{3 a} \\
\mathrm{g} \text { of } \mathbf{3 b}\end{array}$ \\
\hline
\end{tabular}


Table S2. Crystal data and structure refinement for 2.

\begin{tabular}{|c|c|c|}
\hline Identification code & \multicolumn{2}{|c|}{ 22-acetylisocyclocitrinol A } \\
\hline Empirical formula & \multicolumn{2}{|c|}{$\mathrm{C} 27 \mathrm{H} 38 \mathrm{O} 5$} \\
\hline Formula weight & \multicolumn{2}{|l|}{442.57} \\
\hline Temperature & \multicolumn{2}{|l|}{$173(2) \mathrm{K}$} \\
\hline Wavelength & \multicolumn{2}{|l|}{$0.71073 \AA$} \\
\hline Crystal system & \multicolumn{2}{|l|}{ Monoclinic } \\
\hline Space group & \multicolumn{2}{|l|}{$\mathrm{P} 2(1)$} \\
\hline \multirow[t]{3}{*}{ Unit cell dimensions } & $a=10.753(2) \AA$ & $\alpha=90^{\circ}$ \\
\hline & $b=6.5087(15) \AA$ & $\beta=93.235(5)^{\circ}$. \\
\hline & $c=16.864(4) \AA$ & $\gamma=90^{\circ}$ \\
\hline Volume & \multicolumn{2}{|l|}{$1178.4(5) \AA^{3}$} \\
\hline $\mathrm{Z}$ & \multicolumn{2}{|l|}{2} \\
\hline Density (calculated) & \multicolumn{2}{|l|}{$1.247 \mathrm{Mg} / \mathrm{m}^{3}$} \\
\hline Absorption coefficient & \multicolumn{2}{|l|}{$0.084 \mathrm{~mm}^{-1}$} \\
\hline$F(000)$ & \multicolumn{2}{|l|}{480} \\
\hline Crystal size & \multicolumn{2}{|c|}{$0.40 \times 0.30 \times 0.20 \mathrm{~mm}^{3}$} \\
\hline Theta range for data collection & \multicolumn{2}{|c|}{2.42 to $31.68^{\circ}$} \\
\hline Index ranges & \multicolumn{2}{|c|}{$-15<=\mathrm{h}<=15,-9<=\mathrm{k}<=9,-24<=\mathrm{l}<=23$} \\
\hline Reflections collected & \multicolumn{2}{|c|}{11733} \\
\hline Independent reflections & \multicolumn{2}{|c|}{$6914[\mathrm{R}(\mathrm{int})=0.0209]$} \\
\hline Completeness to theta $=31.68^{\circ}$ & \multicolumn{2}{|c|}{$91.8 \%$} \\
\hline Absorption correction & \multicolumn{2}{|l|}{ SADABS } \\
\hline Max. and min. transmission & \multicolumn{2}{|c|}{0.9833 and 0.9671} \\
\hline Refinement method & \multicolumn{2}{|c|}{ Full-matrix least-squares on $\mathrm{F}^{2}$} \\
\hline Data / restraints / parameters & \multicolumn{2}{|c|}{6914 / 1 / 441} \\
\hline Goodness-of-fit on $\mathrm{F}^{2}$ & \multicolumn{2}{|l|}{1.144} \\
\hline Final $R$ indices $[I>2 \operatorname{sigma}(I)]$ & \multicolumn{2}{|c|}{$\mathrm{R} 1=0.0470, \mathrm{wR} 2=0.1141$} \\
\hline $\mathrm{R}$ indices (all data) & \multicolumn{2}{|c|}{$\mathrm{R} 1=0.0516, \mathrm{wR} 2=0.1165$} \\
\hline Absolute structure parameter & \multicolumn{2}{|c|}{$0.1(8)$} \\
\hline Largest diff. peak and hole & \multicolumn{2}{|c|}{0.377 and -0.260 e. $\AA^{-3}$} \\
\hline
\end{tabular}


Table S3. Atomic coordinates ( x 104) and equivalent isotropic displacement parameters $\left(\AA^{2} \mathrm{x} 10^{3}\right)$ for 2. $U(\mathrm{eq})$ is defined as one third of the trace of the orthogonalized $\mathrm{U}^{\mathrm{ij}}$ tensor.

\begin{tabular}{|c|c|c|c|c|}
\hline & $\mathrm{X}$ & $\mathrm{y}$ & $\mathrm{z}$ & $\mathrm{U}(\mathrm{eq})$ \\
\hline $\mathrm{O}(1)$ & $7511(1)$ & 2793(2) & $6174(1)$ & $28(1)$ \\
\hline $\mathrm{O}(2)$ & $9142(1)$ & $-870(2)$ & 8108(1) & $24(1)$ \\
\hline $\mathrm{O}(3)$ & $6330(1)$ & $-1309(2)$ & $12710(1)$ & $27(1)$ \\
\hline $\mathrm{O}(4)$ & $8546(1)$ & $2114(2)$ & $13765(1)$ & $26(1)$ \\
\hline $\mathrm{O}(5)$ & $10352(1)$ & 474(3) & $14008(1)$ & $50(1)$ \\
\hline$C(1)$ & 7644(2) & $4583(2)$ & $8310(1)$ & $20(1)$ \\
\hline $\mathrm{C}(2)$ & $7257(2)$ & $4540(3)$ & $7434(1)$ & $22(1)$ \\
\hline$C(3)$ & 7701(1) & $2585(2)$ & $7020(1)$ & $19(1)$ \\
\hline$C(4)$ & $7010(1)$ & $625(2)$ & $7225(1)$ & $20(1)$ \\
\hline$C(5)$ & $6986(1)$ & $56(2)$ & $8106(1)$ & $18(1)$ \\
\hline$C(6)$ & $8278(1)$ & $-339(2)$ & $8499(1)$ & $17(1)$ \\
\hline$C(7)$ & $8449(1)$ & $-347(2)$ & $9389(1)$ & $17(1)$ \\
\hline $\mathrm{C}(8)$ & $8179(1)$ & $1115(2)$ & $9916(1)$ & $15(1)$ \\
\hline $\mathrm{C}(9)$ & $7667(1)$ & $3236(2)$ & $9698(1)$ & $17(1)$ \\
\hline$C(10)$ & $7183(1)$ & $3320(2)$ & 8841(1) & $17(1)$ \\
\hline $\mathrm{C}(11)$ & $6661(2)$ & $3970(2)$ & $10258(1)$ & $20(1)$ \\
\hline$C(12)$ & $6988(2)$ & $3582(2)$ & $11144(1)$ & $19(1)$ \\
\hline$C(13)$ & $7326(1)$ & $1315(2)$ & 11291(1) & $14(1)$ \\
\hline$C(14)$ & $8473(1)$ & $843(2)$ & $10794(1)$ & $16(1)$ \\
\hline$C(15)$ & $8935(1)$ & $-1239(2)$ & $11112(1)$ & $19(1)$ \\
\hline$C(16)$ & $8737(2)$ & $-1113(3)$ & $12012(1)$ & $22(1)$ \\
\hline$C(17)$ & 7899(1) & $804(2)$ & $12139(1)$ & $16(1)$ \\
\hline $\mathrm{C}(18)$ & $6277(1)$ & $1646(2)$ & 8586(1) & $18(1)$ \\
\hline$C(19)$ & $6233(1)$ & $-101(3)$ & $11038(1)$ & $19(1)$ \\
\hline $\mathrm{C}(20)$ & $7044(1)$ & 543(3) & $12837(1)$ & $19(1)$ \\
\hline $\mathrm{C}(21)$ & $6169(2)$ & $2366(3)$ & $12935(1)$ & $28(1)$ \\
\hline$C(22)$ & $7882(1)$ & 198(3) & $13614(1)$ & 21(1) \\
\hline $\mathrm{C}(23)$ & $7158(2)$ & $-297(3)$ & $14327(1)$ & $24(1)$ \\
\hline $\mathrm{C}(24)$ & $6904(2)$ & $-2177(3)$ & $14559(1)$ & $33(1)$ \\
\hline $\mathrm{C}(25)$ & 6131(3) & $-2675(5)$ & $15252(1)$ & $46(1)$ \\
\hline$C(26)$ & $9777(2)$ & 2039(4) & $13939(1)$ & $32(1)$ \\
\hline $\mathrm{C}(27)$ & $10290(2)$ & $4180(5)$ & $14018(2)$ & $49(1)$ \\
\hline
\end{tabular}


Table S4. Bond lengths $[\AA]$ and angles $\left[{ }^{\circ}\right]$ for 2

\begin{tabular}{|c|c|}
\hline $\mathrm{O}(1)-\mathrm{C}(3)$ & $1.4354(19)$ \\
\hline $\mathrm{O}(2)-\mathrm{C}(6)$ & $1.2197(19)$ \\
\hline $\mathrm{O}(3)-\mathrm{C}(20)$ & $1.4387(19)$ \\
\hline $\mathrm{O}(4)-\mathrm{C}(26)$ & $1.340(2)$ \\
\hline $\mathrm{O}(4)-\mathrm{C}(22)$ & $1.452(2)$ \\
\hline $\mathrm{O}(5)-\mathrm{C}(26)$ & $1.194(3)$ \\
\hline $\mathrm{C}(1)-\mathrm{C}(10)$ & $1.332(2)$ \\
\hline $\mathrm{C}(1)-\mathrm{C}(2)$ & $1.513(2)$ \\
\hline$C(2)-C(3)$ & $1.540(2)$ \\
\hline$C(3)-C(4)$ & $1.526(2)$ \\
\hline$C(4)-C(5)$ & $1.533(2)$ \\
\hline $\mathrm{C}(5)-\mathrm{C}(6)$ & $1.527(2)$ \\
\hline$C(5)-C(18)$ & $1.542(2)$ \\
\hline $\mathrm{C}(6)-\mathrm{C}(7)$ & $1.501(2)$ \\
\hline $\mathrm{C}(7)-\mathrm{C}(8)$ & $1.345(2)$ \\
\hline $\mathrm{C}(8)-\mathrm{C}(14)$ & $1.506(2)$ \\
\hline $\mathrm{C}(8)-\mathrm{C}(9)$ & $1.523(2)$ \\
\hline $\mathrm{C}(9)-\mathrm{C}(10)$ & $1.509(2)$ \\
\hline $\mathrm{C}(9)-\mathrm{C}(11)$ & $1.551(2)$ \\
\hline $\mathrm{C}(10)-\mathrm{C}(18)$ & $1.509(2)$ \\
\hline $\mathrm{C}(11)-\mathrm{C}(12)$ & $1.535(2)$ \\
\hline $\mathrm{C}(12)-\mathrm{C}(13)$ & $1.537(2)$ \\
\hline C(13)-C(19) & $1.535(2)$ \\
\hline $\mathrm{C}(13)-\mathrm{C}(17)$ & $1.561(2)$ \\
\hline C(13)-C(14) & $1.560(2)$ \\
\hline $\mathrm{C}(14)-\mathrm{C}(15)$ & $1.530(2)$ \\
\hline $\mathrm{C}(15)-\mathrm{C}(16)$ & $1.547(2)$ \\
\hline $\mathrm{C}(16)-\mathrm{C}(17)$ & $1.561(2)$ \\
\hline $\mathrm{C}(17)-\mathrm{C}(20)$ & $1.543(2)$ \\
\hline $\mathrm{C}(20)-\mathrm{C}(21)$ & $1.529(2)$ \\
\hline $\mathrm{C}(20)-\mathrm{C}(22)$ & $1.563(2)$ \\
\hline $\mathrm{C}(22)-\mathrm{C}(23)$ & $1.503(2)$ \\
\hline $\mathrm{C}(23)-\mathrm{C}(24)$ & $1.318(3)$ \\
\hline $\mathrm{C}(24)-\mathrm{C}(25)$ & $1.507(3)$ \\
\hline$C(26)-C(27)$ & $1.502(3)$ \\
\hline $\mathrm{C}(26)-\mathrm{O}(4)-\mathrm{C}(22)$ & $118.50(15)$ \\
\hline $\mathrm{C}(10)-\mathrm{C}(1)-\mathrm{C}(2)$ & $123.60(14)$ \\
\hline $\mathrm{C}(1)-\mathrm{C}(2)-\mathrm{C}(3)$ & $112.52(13)$ \\
\hline $\mathrm{O}(1)-\mathrm{C}(3)-\mathrm{C}(4)$ & $105.08(12)$ \\
\hline $\mathrm{O}(1)-\mathrm{C}(3)-\mathrm{C}(2)$ & 109.99(12) \\
\hline $\mathrm{C}(4)-\mathrm{C}(3)-\mathrm{C}(2)$ & $114.88(13)$ \\
\hline$C(3)-C(4)-C(5)$ & $117.16(12)$ \\
\hline $\mathrm{C}(6)-\mathrm{C}(5)-\mathrm{C}(4)$ & $113.38(12)$ \\
\hline $\mathrm{C}(6)-\mathrm{C}(5)-\mathrm{C}(18)$ & $110.50(12)$ \\
\hline$C(4)-C(5)-C(18)$ & $112.51(12)$ \\
\hline $\mathrm{O}(2)-\mathrm{C}(6)-\mathrm{C}(7)$ & $119.10(13)$ \\
\hline $\mathrm{O}(2)-\mathrm{C}(6)-\mathrm{C}(5)$ & $120.95(14)$ \\
\hline$C(7)-C(6)-C(5)$ & $119.45(12)$ \\
\hline $\mathrm{C}(8)-\mathrm{C}(7)-\mathrm{C}(6)$ & $129.73(13)$ \\
\hline $\mathrm{C}(7)-\mathrm{C}(8)-\mathrm{C}(14)$ & $121.66(13)$ \\
\hline $\mathrm{C}(7)-\mathrm{C}(8)-\mathrm{C}(9)$ & $124.77(13)$ \\
\hline $\mathrm{C}(14)-\mathrm{C}(8)-\mathrm{C}(9)$ & $113.36(12)$ \\
\hline $\mathrm{C}(10)-\mathrm{C}(9)-\mathrm{C}(8)$ & $111.35(12)$ \\
\hline$C(10)-C(9)-C(11)$ & $110.92(12)$ \\
\hline$C(8)-C(9)-C(11)$ & $112.79(12)$ \\
\hline $\mathrm{C}(1)-\mathrm{C}(10)-\mathrm{C}(9)$ & $123.04(14)$ \\
\hline$C(1)-C(10)-C(18)$ & $120.86(13)$ \\
\hline
\end{tabular}




$\begin{array}{lc}\mathrm{C}(9)-\mathrm{C}(10)-\mathrm{C}(18) & 115.43(12) \\ \mathrm{C}(12)-\mathrm{C}(11)-\mathrm{C}(9) & 114.33(12) \\ \mathrm{C}(11)-\mathrm{C}(12)-\mathrm{C}(13) & 110.76(12) \\ \mathrm{C}(19)-\mathrm{C}(13)-\mathrm{C}(12) & 111.16(12) \\ \mathrm{C}(19)-\mathrm{C}(13)-\mathrm{C}(17) & 112.56(12) \\ \mathrm{C}(12)-\mathrm{C}(13)-\mathrm{C}(17) & 115.50(12) \\ \mathrm{C}(19)-\mathrm{C}(13)-\mathrm{C}(14) & 110.35(12) \\ \mathrm{C}(12)-\mathrm{C}(13)-\mathrm{C}(14) & 106.81(12) \\ \mathrm{C}(17)-\mathrm{C}(13)-\mathrm{C}(14) & 99.63(11) \\ \mathrm{C}(8)-\mathrm{C}(14)-\mathrm{C}(15) & 119.62(12) \\ \mathrm{C}(8)-\mathrm{C}(14)-\mathrm{C}(13) & 112.11(11) \\ \mathrm{C}(15)-\mathrm{C}(14)-\mathrm{C}(13) & 103.73(12) \\ \mathrm{C}(14)-\mathrm{C}(15)-\mathrm{C}(16) & 103.67(12) \\ \mathrm{C}(15)-\mathrm{C}(16)-\mathrm{C}(17) & 106.88(12) \\ \mathrm{C}(20)-\mathrm{C}(17)-\mathrm{C}(13) & 120.06(12) \\ \mathrm{C}(20)-\mathrm{C}(17)-\mathrm{C}(16) & 112.96(12) \\ \mathrm{C}(13)-\mathrm{C}(17)-\mathrm{C}(16) & 104.18(11) \\ \mathrm{C}(10)-\mathrm{C}(18)-\mathrm{C}(5) & 107.65(12) \\ \mathrm{O}(3)-\mathrm{C}(20)-\mathrm{C}(21) & 109.83(13) \\ \mathrm{O}(3)-\mathrm{C}(20)-\mathrm{C}(17) & 108.35(12) \\ \mathrm{C}(21)-\mathrm{C}(20)-\mathrm{C}(17) & 113.15(13) \\ \mathrm{O}(3)-\mathrm{C}(20)-\mathrm{C}(22) & 106.41(12) \\ \mathrm{C}(21)-\mathrm{C}(20)-\mathrm{C}(22) & 110.51(13) \\ \mathrm{C}(17)-\mathrm{C}(20)-\mathrm{C}(22) & 108.34(11) \\ \mathrm{O}(4)-\mathrm{C}(22)-\mathrm{C}(23) & 108.39(13) \\ \mathrm{O}(4)-\mathrm{C}(22)-\mathrm{C}(20) & 106.17(12) \\ \mathrm{C}(23)-\mathrm{C}(22)-\mathrm{C}(20) & 113.62(12) \\ \mathrm{C}(24)-\mathrm{C}(23)-\mathrm{C}(22) & 124.19(17) \\ \mathrm{C}(23)-\mathrm{C}(24)-\mathrm{C}(25) & 124.2(2) \\ \mathrm{O}(5)-\mathrm{C}(26)-\mathrm{O}(4) & 123.5(2) \\ \mathrm{O}(5)-\mathrm{C}(26)-\mathrm{C}(27) & 126.64(19) \\ \mathrm{O}(4)-\mathrm{C}(26)-\mathrm{C}(27) & 109.8(2) \\ & \\ & \end{array}$

Symmetry transformations used to generate equivalent atoms: 
Table S5. Anisotropic displacement parameters $\left(\AA^{2} \mathrm{x} 10^{3}\right)$ for 2 . The anisotropic displacement factor exponent takes the form: $-2 \pi^{2}\left[h^{2} a^{* 2} U^{11}+\ldots+2 h k a^{*} b^{*} U^{12}\right]$

\begin{tabular}{|c|c|c|c|c|c|c|}
\hline & $\mathrm{U}^{11}$ & $\mathrm{U}^{22}$ & $\mathrm{U}^{33}$ & $\mathrm{U}^{23}$ & $\mathrm{U}^{13}$ & $\mathrm{U}^{12}$ \\
\hline $\mathrm{O}(1)$ & $38(1)$ & $27(1)$ & $18(1)$ & $1(1)$ & $2(1)$ & $-2(1)$ \\
\hline $\mathrm{O}(2)$ & $25(1)$ & $25(1)$ & $22(1)$ & 1(1) & $6(1)$ & $7(1)$ \\
\hline $\mathrm{O}(3)$ & $26(1)$ & $35(1)$ & $21(1)$ & $2(1)$ & $1(1)$ & $-14(1)$ \\
\hline $\mathrm{O}(4)$ & $22(1)$ & $32(1)$ & $24(1)$ & $-2(1)$ & $-2(1)$ & $-6(1)$ \\
\hline $\mathrm{O}(5)$ & $24(1)$ & $72(1)$ & $54(1)$ & $-10(1)$ & $-4(1)$ & $7(1)$ \\
\hline$C(1)$ & $26(1)$ & $11(1)$ & $21(1)$ & $-1(1)$ & $1(1)$ & 2(1) \\
\hline $\mathrm{C}(2)$ & $31(1)$ & 17(1) & $19(1)$ & $2(1)$ & $0(1)$ & $4(1)$ \\
\hline $\mathrm{C}(3)$ & $21(1)$ & $18(1)$ & $19(1)$ & $0(1)$ & 1(1) & $2(1)$ \\
\hline$C(4)$ & $23(1)$ & $17(1)$ & $19(1)$ & $-2(1)$ & 1(1) & $-1(1)$ \\
\hline$C(5)$ & $18(1)$ & $13(1)$ & $21(1)$ & $-2(1)$ & 1(1) & $-1(1)$ \\
\hline$C(6)$ & $21(1)$ & $10(1)$ & $22(1)$ & $1(1)$ & $2(1)$ & $1(1)$ \\
\hline $\mathrm{C}(7)$ & $17(1)$ & $14(1)$ & 21(1) & $2(1)$ & $2(1)$ & $2(1)$ \\
\hline $\mathrm{C}(8)$ & $12(1)$ & $14(1)$ & $18(1)$ & 1(1) & $2(1)$ & $-1(1)$ \\
\hline $\mathrm{C}(9)$ & $18(1)$ & $12(1)$ & $19(1)$ & $-1(1)$ & $2(1)$ & $0(1)$ \\
\hline$C(10)$ & $18(1)$ & $12(1)$ & $20(1)$ & $-3(1)$ & 1(1) & $6(1)$ \\
\hline $\mathrm{C}(11)$ & $26(1)$ & $16(1)$ & 19(1) & $-1(1)$ & $2(1)$ & $7(1)$ \\
\hline$C(12)$ & $24(1)$ & $15(1)$ & $18(1)$ & $-2(1)$ & $3(1)$ & $4(1)$ \\
\hline$C(13)$ & $14(1)$ & $14(1)$ & $16(1)$ & $-1(1)$ & 1(1) & $0(1)$ \\
\hline $\mathrm{C}(14)$ & $13(1)$ & $16(1)$ & $18(1)$ & $1(1)$ & $2(1)$ & $0(1)$ \\
\hline$C(15)$ & $18(1)$ & $20(1)$ & $20(1)$ & 1(1) & $3(1)$ & $5(1)$ \\
\hline$C(16)$ & $23(1)$ & $24(1)$ & $19(1)$ & $4(1)$ & 1(1) & $7(1)$ \\
\hline$C(17)$ & $13(1)$ & $19(1)$ & $17(1)$ & $-1(1)$ & $2(1)$ & $-1(1)$ \\
\hline $\mathrm{C}(18)$ & $16(1)$ & $18(1)$ & $20(1)$ & $-1(1)$ & $2(1)$ & $1(1)$ \\
\hline$C(19)$ & $15(1)$ & $23(1)$ & $18(1)$ & $-1(1)$ & $0(1)$ & $-5(1)$ \\
\hline$C(20)$ & $15(1)$ & $24(1)$ & $18(1)$ & $0(1)$ & $0(1)$ & $-3(1)$ \\
\hline $\mathrm{C}(21)$ & $22(1)$ & $39(1)$ & $23(1)$ & $1(1)$ & $4(1)$ & $8(1)$ \\
\hline$C(22)$ & $17(1)$ & $27(1)$ & $18(1)$ & $-1(1)$ & $0(1)$ & $-1(1)$ \\
\hline$C(23)$ & $20(1)$ & $34(1)$ & $18(1)$ & $1(1)$ & 1(1) & $1(1)$ \\
\hline $\mathrm{C}(24)$ & $36(1)$ & $36(1)$ & $28(1)$ & $4(1)$ & $5(1)$ & $-3(1)$ \\
\hline$C(25)$ & $54(1)$ & $49(1)$ & $34(1)$ & $13(1)$ & $12(1)$ & $-6(1)$ \\
\hline$C(26)$ & $21(1)$ & $57(1)$ & $18(1)$ & $-6(1)$ & $3(1)$ & $-8(1)$ \\
\hline $\mathrm{C}(27)$ & $39(1)$ & $68(2)$ & $38(1)$ & $-6(1)$ & $0(1)$ & $-30(1)$ \\
\hline
\end{tabular}


Table S6. Hydrogen coordinates ( x 10 $)$ and isotropic displacement parameters $\left(\AA^{2} \mathrm{x} 10^{3}\right)$ for 2 .

\begin{tabular}{|c|c|c|c|c|}
\hline & $\mathrm{x}$ & $\mathrm{y}$ & $\mathrm{z}$ & $\mathrm{U}(\mathrm{eq})$ \\
\hline $\mathrm{H}(1 \mathrm{O})$ & $8070(20)$ & $3490(40)$ & $6050(14)$ & $28(6)$ \\
\hline $\mathrm{H}(3 \mathrm{O})$ & $6040(20)$ & $-1440(50)$ & $13080(17)$ & $41(7)$ \\
\hline $\mathrm{H}(1)$ & $8270(20)$ & $5460(40)$ & $8484(13)$ & $26(5)$ \\
\hline $\mathrm{H}(2 \mathrm{~B})$ & $7616(18)$ & $5680(40)$ & $7166(13)$ & $20(5)$ \\
\hline $\mathrm{H}(2 \mathrm{~A})$ & $6347(19)$ & $4610(30)$ & 7351(11) & $19(5)$ \\
\hline $\mathrm{H}(3)$ & $8587(17)$ & $2430(30)$ & $7152(11)$ & $16(4)$ \\
\hline $\mathrm{H}(4 \mathrm{~B})$ & 7312(19) & $-530(40)$ & $6926(12)$ & $22(5)$ \\
\hline $\mathrm{H}(4 \mathrm{~A})$ & 6133(19) & $760(30)$ & $7009(12)$ & $21(5)$ \\
\hline $\mathrm{H}(5)$ & $6577(16)$ & $-1210(30)$ & $8142(11)$ & $14(4)$ \\
\hline $\mathrm{H}(7)$ & $8836(19)$ & $-1440(40)$ & $9552(13)$ & $26(5)$ \\
\hline $\mathrm{H}(9)$ & $8341(18)$ & $4180(30)$ & $9765(11)$ & $17(5)$ \\
\hline $\mathrm{H}(11 \mathrm{~B})$ & $5896(17)$ & $3400(30)$ & $10102(11)$ & $10(4)$ \\
\hline $\mathrm{H}(11 \mathrm{~A})$ & $6576(19)$ & $5320(40)$ & $10165(13)$ & $23(5)$ \\
\hline $\mathrm{H}(12 \mathrm{~B})$ & $6273(18)$ & $3990(30)$ & $11456(11)$ & $17(4)$ \\
\hline $\mathrm{H}(12 \mathrm{~A})$ & $7720(20)$ & $4340(40)$ & $11323(12)$ & $25(5)$ \\
\hline $\mathrm{H}(14)$ & $9060(20)$ & $1830(30)$ & $10955(12)$ & $20(5)$ \\
\hline $\mathrm{H}(15 \mathrm{~B})$ & $8450(20)$ & $-2310(40)$ & $10884(13)$ & $28(5)$ \\
\hline $\mathrm{H}(15 \mathrm{~A})$ & $9750(20)$ & $-1530(40)$ & 10981(13) & $33(6)$ \\
\hline $\mathrm{H}(16 \mathrm{~B})$ & $8340(20)$ & $-2420(40)$ & $12177(14)$ & $36(6)$ \\
\hline $\mathrm{H}(16 \mathrm{~A})$ & $9513(17)$ & $-970(30)$ & $12314(11)$ & $14(4)$ \\
\hline $\mathrm{H}(17)$ & $8397(17)$ & $1900(30)$ & $12248(11)$ & $15(5)$ \\
\hline $\mathrm{H}(18 \mathrm{~B})$ & $5612(18)$ & $2130(30)$ & $8240(12)$ & $20(5)$ \\
\hline $\mathrm{H}(18 \mathrm{~A})$ & $5890(20)$ & $970(40)$ & $9042(13)$ & $25(5)$ \\
\hline $\mathrm{H}(19 \mathrm{C})$ & $6079(19)$ & $-90(30)$ & $10502(13)$ & $21(5)$ \\
\hline $\mathrm{H}(19 \mathrm{~B})$ & $6448(18)$ & $-1440(40)$ & $11192(12)$ & $21(5)$ \\
\hline $\mathrm{H}(19 \mathrm{~A})$ & $5431(18)$ & $370(30)$ & $11276(12)$ & $20(5)$ \\
\hline $\mathrm{H}(21 \mathrm{C})$ & $5559(18)$ & $2350(40)$ & $12504(12)$ & $19(5)$ \\
\hline $\mathrm{H}(21 \mathrm{~B})$ & $5740(20)$ & $2270(40)$ & $13422(15)$ & $38(6)$ \\
\hline $\mathrm{H}(21 \mathrm{~A})$ & $6645(19)$ & $3580(40)$ & $12960(13)$ & $25(5)$ \\
\hline $\mathrm{H}(26)$ & $8473(17)$ & $-820(30)$ & $13545(11)$ & $14(4)$ \\
\hline $\mathrm{H}(23)$ & $6860(20)$ & $880(40)$ & $14622(14)$ & $31(6)$ \\
\hline $\mathrm{H}(24)$ & $7220(30)$ & $-3280(50)$ & $14259(17)$ & $47(7)$ \\
\hline $\mathrm{H}(25 \mathrm{C})$ & $6450(30)$ & $-3730(60)$ & $15580(20)$ & $62(9)$ \\
\hline $\mathrm{H}(25 \mathrm{~B})$ & $5440(30)$ & $-3470(50)$ & $15040(20)$ & $63(10)$ \\
\hline $\mathrm{H}(25 \mathrm{~A})$ & $5920(30)$ & $-1510(60)$ & $15534(19)$ & $62(9)$ \\
\hline $\mathrm{H}(27 \mathrm{C})$ & $11180(30)$ & $4170(60)$ & $14220(19)$ & $72(10)$ \\
\hline $\mathrm{H}(27 \mathrm{~B})$ & $9810(40)$ & $5060(80)$ & $14310(30)$ & $108(15)$ \\
\hline $\mathrm{H}(27 \mathrm{~A})$ & $10270(30)$ & $4740(60)$ & $13520(20)$ & $80(11)$ \\
\hline
\end{tabular}

\footnotetext{
${ }^{1}$ Varoglu, M.; Crews, P. J. Nat. Prod. 2000, 63, 41-43.

2 SMART \& SAINT Software Reference manuals, version 5.042; Bruker Analytical X-ray Systems. Madison WI, 1998.

3 Sheldrick G.M. SADABS, a software for empirical absorption correction; University of Gottingen, Germany, 2000 .
} 
${ }^{4}$ Sheldrick G.M, SHELXTL Reference Manual, version 5.1; Bruker Analytical X-ray Systems. Madison WI, 1997. 